\title{
Benefits of the multi-modality formulation in hydrogen supply chain modelling
}

\author{
Federico Parolin ${ }^{1}$, Paolo Colbertaldo ${ }^{1,}$, and Stefano Campanari ${ }^{1}$ \\ ${ }^{1}$ Group of Energy Conversion Systems (GECOS), Department of Energy, Politecnico di Milano, 20156 Milan, Italy
}

\begin{abstract}
Hydrogen is recognized as a key element of future low-carbon energy systems. For proper integration, an adequate delivery infrastructure will be required, to be deployed in parallel to the electric grid and the gas network. This work adopts an optimization model to support the design of a future hydrogen delivery infrastructure, considering production, storage, and transport up to demand points. The model includes two production technologies, i.e., steam reforming with carbon capture and PV-fed electrolysis systems, and three transport modalities, i.e., pipelines, compressed hydrogen trucks, and liquid hydrogen trucks. This study compares a multi-modality formulation, in which the different transport technologies are simultaneously employed and their selection is optimized, with a mono-modality formulation, in which a single transport technology is considered. The assessment looks at the regional case study of Lombardy in Italy, considering a long-term scenario in which an extensive hydrogen supply chain is developed to supply hydrogen for clean mobility. Results show that the multi-modality infrastructure provides significant cost benefits, yielding an average cost of hydrogen that is up to $11 \%$ lower than a mono-modality configuration.
\end{abstract}

\section{Introduction}

With the European Green Deal, the European Union has committed to reach carbon neutrality by 2050 , calling all the energy-consuming sectors to a paradigm-shifting transition towards decarbonization [1].

The development of a hydrogen economy is expected to be one of the pillars of the energy transition, as hydrogen can be employed in a variety of applications, allowing to abate emissions in diverse sectors [2]. This energy vector appears particularly promising for the decarbonization of the transport sector, which alone accounts for more than $30 \%$ of $\mathrm{CO}_{2}$ emissions in Europe [3]. Hydrogen-powered fuel cell electric vehicles (FCEVs) ensure zero tailpipe emissions, offering higher mileage and faster refuelling if compared to battery electric vehicles (BEVs). In addition to passenger cars, FCEVs are highly suitable for buses and heavy-duty transport, where weight constraints are relevant and payload reductions must be avoided [4].

The realization of an extensive delivery infrastructure is of paramount importance to assist the development of a hydrogen-based clean mobility, as the lack of a distributed supply chain currently limits the access to this energy vector. Accordingly, the topic of the hydrogen supply chain (HSC) has drawn increasing interest in the scientific community, and different authors developed modelling tools to design and analyse the hydrogen infrastructure. The HSC modelling features an inherent complexity, as each stage (production, transport, storage, and consumption) may take place with competing alternatives, each coming with advantages and drawbacks, as well as different costs and industrial maturity.

The existing literature features a variety of approaches to deal with such complexity, and each work addresses different aspects with distinct assumptions. The majority of the studies consists of mono-objective optimization models, aimed at minimising the total cost of the infrastructure [5]-[18]. A few works deal with multiobjective optimization, in which the cost minimisation is combined with environmental, financial, or safety concerns [19]-[25], while others focus on parametric analyses, to compare alternative infrastructure configurations [26], [27].

Many works analyse a snapshot of the infrastructure, i.e., a representative steady-state condition with timeinvariant quantities [5], [6], [9]-[11], [15], [24], [26], [27], thus sizing the HSC according to the condition identified as the most stressful moment throughout the year, e.g., in terms of highest demand and/or lowest production. While this approach allows to reduce the computational complexity of the model, it fails to track the optimal design and operation of storage units, which is paramount when dealing with hydrogen production from intermittent renewable energy sources (RES). An alternative approach is to consider a year-long analysis, tracking the variation of quantities according to a certain time resolution [17], [18], thus obtaining a more accurate description of the HSC, at the expenses of a higher model complexity. A further alternative is to analyse the evolution of the infrastructure over a long time horizon through a multi-period analysis, which consists of dividing the considered timeframe in intervals of equal

\footnotetext{
${ }^{*}$ Corresponding author: paolo.colbertaldo@polimi.it
} 
duration, treated similarly to snapshots [7], [14], [16], [20]-[23], [25].

The spatial modelling of the HSC is often addressed by adopting schematized networks with a restricted number of points and connections to limit the number of variables and equations [7], [10], [14]-[17], [20], [21], [23]-[25], [27]. A more accurate, yet more computationally demanding, approach is to define the candidate infrastructure network on the basis of actual spatial data through a Geographic Information System (GIS), thereby introducing territorial constraints in the model.

A crucial aspect in the HSC modelling is the multiplicity of hydrogen transport modalities. Since these are not alternative, but rather competing, it would be beneficial to optimize also the selection of the transport technologies for each stage of the supply chain in a multimodality formulation. However, since this approach significantly increases the model complexity and computational requirements, it is featured in a limited number of studies [7], [8], [10], [11], [14], [16], [19]-[21], [23], [24], which typically introduce other simplifying modelling assumptions, whereas the majority considers a single transport modality at a time in mono-modality formulations.

This work adopts a spatially and temporally resolved mixed integer linear programming (MILP) model to optimize the design and operation of a hydrogen infrastructure to supply a network of hydrogen refuelling stations (HRS) [28]. The model includes all the stages of the HSC (production, transport, storage, and demand) and optimizes the infrastructure taking into account the candidate HSC networks based on GIS data, considering a year-long analysis with daily resolution. Two production technologies are considered in this analysis (although the model could also include a larger variety of options), (i) steam methane reforming equipped with carbon capture and (ii) electrolysis fed by dedicated solar photovoltaic (PV) plants. The included transport modalities are hydrogen pipelines, compressed hydrogen trucks, and liquid hydrogen trucks, and a multi-modality formulation is developed to optimize their selection. The adopted tool also allows to impose the selection parameters at start, in order to perform mono-modality simulations, i.e., considering only one transport mode.
After presenting an overview of the model formulation, the article focuses on the comparison between the multi- and the mono-modality formulations, according to the results obtained from the model applications to the case study of the Italian region of Lombardy, considering a long-term scenario in which a $25 \%$ FCEV stock share is assumed among passenger cars.

Compared to other works in the existing literature that presented a multi-modality formulation, the added value of the adopted model is the combination of the multimodality formulation, the detailed description of the HSC pathways, the inclusion of territorial constraints through GIS data, and the time-variant analysis, as these characteristics are not simultaneously featured by any of the studies identified in the literature survey.

\section{Problem statement}

The adopted model minimizes the total cost of the infrastructure. The objective function is set equal to the average cost of hydrogen delivered to demand points, considering investment and operational costs for hydrogen production, conditioning, transport, and storage:

$$
f_{o b}=\min \left\{\frac{\sum_{i} C A P E X_{i} \cdot C R F_{i}+\sum_{i} O P E X_{i}}{\sum_{n} \sum_{t} q_{d e m}^{n, t}}\right\}
$$

Figure 1 summarizes the technological options considered for each stage of the HSC. Hydrogen production in the model can exploit two different technologies. The first option is steam methane reforming equipped with carbon capture and storage (SMR-CCS), which ensures a flexible production of blue hydrogen within the plant nominal capacity. The second option is electrolysis (EL) fed by dedicated PV plants (PV-EL), which produces green hydrogen with a constrained output that depends on the availability of solar radiation. The purchase of grid electricity is allowed to partially compensate this issue, while revenues can be obtained from the sale of surplus electricity that may result due to the PV vs. EL nominal capacity ratio or in periods of low hydrogen demand and large availability of solar radiation.

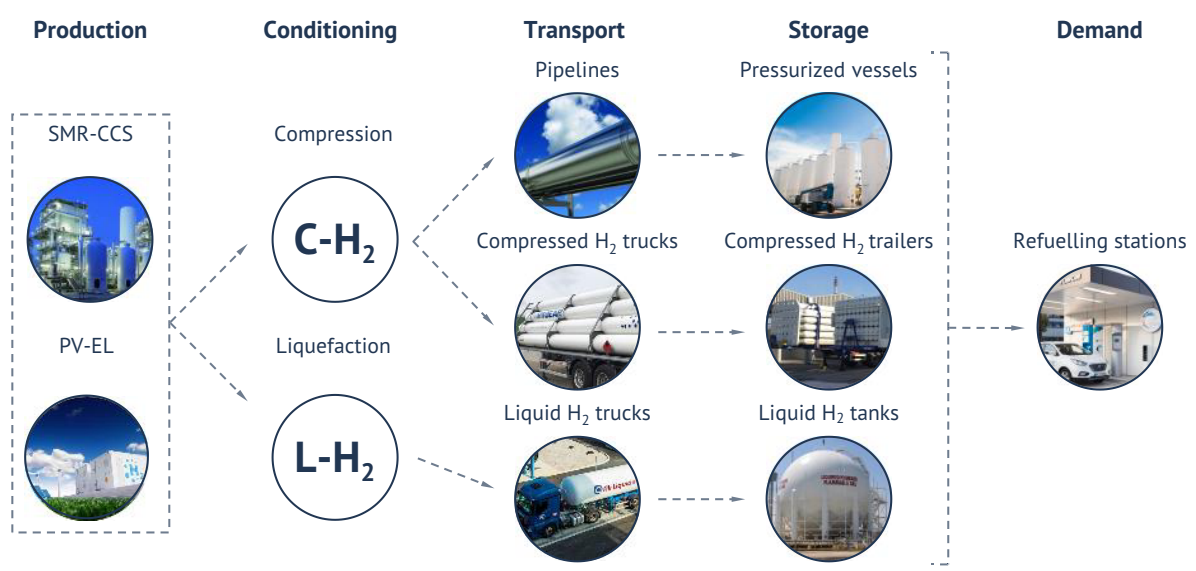

Figure 1. Scheme of the HSC stages, depicting the included technological options. 
Conditioning to compressed or liquid hydrogen is performed at production sites, according to the subsequent transport technology. In particular, hydrogen can be delivered as a gas via pipeline (GP), as a gas in a compressed hydrogen truck (GT), or as a liquid in a cryogenic truck (LT). Depending on the employed modality (see Figure 1), hydrogen is stored in pressurized vessels at 160 bar, in the trailer vessels themselves, or in cryogenic insulated tanks.

The end point of hydrogen pathways are the HRSs. Each HRS can be supplied exclusively via one of the three included transport modalities, and the selection is optimized. The internal station structure is not investigated and the installation costs are left out of the boundaries of the analysis since they do not vary significantly between the different types of stations [9]. Storage needs at HRSs are instead included, setting an upper boundary to the installed capacity depending on the maximum daily demand of each node, since the space availability of HRSs is typically constrained. The daily demand profiles for each station are provided as input to the model, and they vary depending on the time of year, whereas the annual demand depends on the station location.

\section{Model structure}

The HSC is modelled as a network of nodes and edges. In order to model the infrastructure components represented in Figure 1, the network topology comprises four node types: production, demand, intermediate storage, and transit. Except for transit nodes, all node types feature a storage section and a virtual sub-node, which is responsible for the connection with the network edges. According to this approach, the node mass balance is defined by two equations, one for the storage section, and one for the virtual sub-node. In a general form, the equations for each node $n$ and each transport modality $m$, at each time step $t$ are:

$$
\left\{\begin{array}{c}
Q_{s}^{n, m, t+1}=Q_{s}^{n, m, t}+\left(q_{i n, s}^{n, m, t}-q_{\text {out }, s}^{n, m}-\xi_{d e m}^{m} q_{d e m}^{n, t}\right) \cdot \Delta t \cdot N_{t d} \\
Y^{n, m} \cdot q_{e d g}^{e, m, t}=q_{o u t, s}^{n, m, t}-q_{i n, s}^{n, m, t}+\xi_{p r d}^{m} q_{p r d}^{m, t}
\end{array}\right.
$$

where $q_{\text {dem }}$ is the hydrogen demand, $q_{\text {prd }}$ is the production flow, $q_{e d g}^{e}$ is the flow along edge $e, Q_{s}$ is the storage content, $q_{i n, s}$ and $q_{o u t, s}$ are the inlet and outlet storage flows, $\xi_{\text {dem }}$ and $\xi_{\text {prd }}$ are equal to 1 for demand and production nodes, respectively, and zero otherwise, and $Y$ is the incidence matrix of the graph representative of the transport network.

The terms $\Delta t$ and $N_{t d}$ in Eq. (2) are related to the model timescale and deserve a separate discussion. The model considers the infrastructure operation over a yearlong timeframe, and, since the quantities are tracked according to a daily resolution, the parameter $\Delta t$ is equal to 1 day. In order to limit the number of variables and equations, the typical day approach is followed. This consists of identifying a set of typical days, each representative of a period of the year, and to repeat them identically for a certain number of times, in terms of demand, production, and flows, up to representing the entire year. In this analysis, 52 typical days are identified, and each of them is repeated $N_{t d}=7$ times to form the 52 weeks of the year. The advantage of this approach is that it allows to substantially reduce the computational complexity while preserving a sufficient level of detail. The intra-week variation of the demand is neglected, whereas the year-long fluctuation of PV generation is preserved.

Figure 2 outlines the high level representation of the HSC in the model. Road and pipeline delivery occur on two parallel graphs, while production and demand nodes are unique and shared between the two networks. In particular, the production flow is injected in the respective graph through conditioning, after which variables become separated, according to the transport modality. As can be noted from Figure 2, intermediate storage is not envisaged for compressed hydrogen trucks, as it is preferable to store the trailers at production sites and transport them to demand points when required than deliver them to an intermediate hub and move them again later.

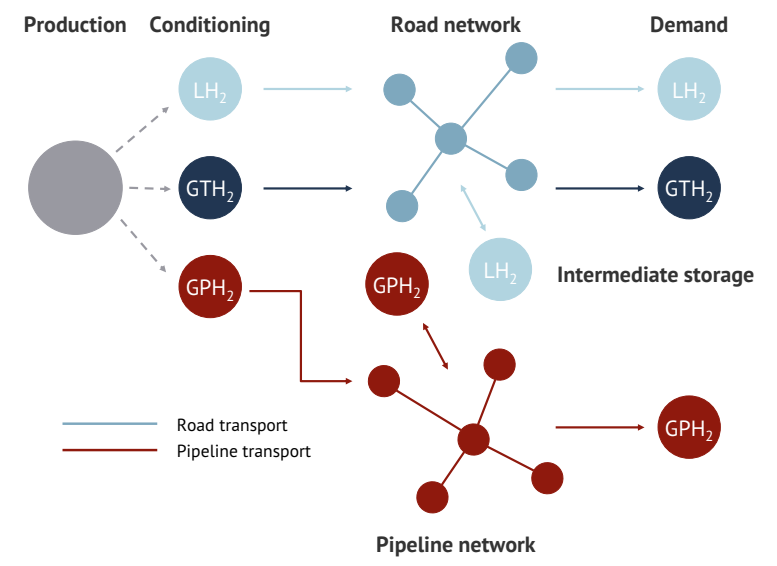

Figure 2. High level representation of the HSC as structured in the model.

Territorial constraints are included by building the candidate transport networks from GIS spatial data, which are available as shapefiles [29]. Candidate truck routes run along the existing road infrastructure, under the hypothesis that it is not saturated, while the current railroad network is adopted as candidate example of graph for pipeline routes, considering it conceptually suitable as it consists of pathways where rights of way already exist and that exclude forbidden or implausible areas. A more plausible alternative could be the current natural gas grid network; however, the access to detailed spatial information on pipeline routes is restricted. At any rate, the main aim in this analysis is to define a candidate network for hydrogen transport via pipeline that differs from the road infrastructure, includes a lower number of edges, and involves areas where infrastructural intervention already occurred. The road and railroad shapefiles are converted into directed graphs by means of dedicated Matlab $^{\circledR}$ functions, which also allow to add production and demand nodes by connecting them to the nearest nodes of the graph. To reduce the size and complexity of the model, the resulting networks are then simplified by maintaining only the transit nodes that host 
multiple intersections of edges. Furthermore, the minimum spanning tree algorithm is applied to extract the subset of edges that yields the minimum network extent, accepting the risk of losing some potentially usable pathways to improve the computational tractability of the model.

To complete the model presentation, the HSC optimization problem is here summarized. The required input data are:

- the set of available hydrogen production, storage, and transport technologies, together with their techno-economic data;

- the topology of the candidate transport networks, the position of production sites, potential intermediate storage hubs, and demand points;

- $\quad$ the demand profiles of HRSs;

- the upper boundaries of production and storage nominal capacities;

- the PV electricity generation profiles and the PVEL nominal capacity ratio.

Then, the infrastructure is designed by the model, while solving also the year-long operation, by optimizing the following:

- the employed production, transport, and storage technologies;

- the installed production capacities;

- the installed storage capacities at production, demand, and intermediate hub nodes;

- the exploited transport pathways and modalities, and the delivered quantities;

- the hydrogen flows for each component of the HSC.

As previously discussed, the analysis presented in this work focuses on the comparison between the mono- and multi-modality formulations. Accordingly, although the model was developed following a multi-modality formulation, its implementation allows to perform also mono-modality simulations, by transforming the variables of the excluded modalities into parameters before running the model.

\section{Case study: assumptions and data}

For the purpose of comparing the mentioned mono/multi-modality formulations, the model is applied to the regional case study of Lombardy in Italy, considering a long-term scenario (2050) in which FCEVs account for $25 \%$ of the passenger car stock share in the country, with small deviations among provinces based on population, population density, vehicle ownership rate, and income per capita [30]. Note that this assumption could be turned into an equivalent distributed consumption e.g. of heavyload trucks or other hydrogen demands, without changing the validity of the example.

The position of HRSs is identified assuming that $10 \%$ of existing gasoline and diesel refuelling stations (i.e., 366 stations) will host hydrogen refuelling, selecting them to guarantee an even spatial presence. Hydrogen demand is computed for each province and distributed uniformly among the stations, which feature an annual demand in the range $0.2-1.1 \mathrm{kt} / \mathrm{y}$, leading to a total of $145 \mathrm{kt} / \mathrm{y}$ in the region.

As far as production is concerned, SMR-CCS plants are assumed to be sited at the existing refineries of ENI Sannazzaro and MOL Mantova [31], while the centroid of each province is selected as candidate location for a centralized PV-EL facility. In terms of maximum capacity, a cap is introduced for (i) the upper boundary for SMR-CCS, which is set to $20 \%$ of today's refinery hydrogen production, and (ii) the dedicated PV capacity in each province, which cannot exceed 5 times the installed PV capacity in 2018 , thus also preserving the proportion among provinces. In the case of PV-EL systems, the hydrogen production is bounded in terms of daily production via an intra-hour analysis that assumes a PV-EL capacity ratio equal to 2 [32].

To complete the networks, 15 candidate intermediate storage sites are identified through a random extraction among transit nodes for both the pipeline and the road delivery graph, considering a minimum distance of $20 \mathrm{~km}$ between them to obtain a homogeneous distribution. This set of nodes is supplied as input to the model, which optimizes the installed capacities and the actual use.

The main techno-economic data are reported in Table 1. In order to be consistent with the assessment of a longterm scenario, values are selected considering available projections or optimistic values among short- and medium- term estimates. All costs have been reported to $€_{2019}$ through inflationary adjustments for both the EU [33] and the US [34].

Table 1. Main techno-economic data.

\begin{tabular}{|c|c|c|c|}
\hline Parameter & Value & Unit & Reference \\
\hline EL CAPEX $^{*}$ & 580 & $€ / \mathrm{kW}_{\mathrm{e}}$ & [35] \\
\hline EL consumption & 49 & $\mathrm{kWh}_{\mathrm{e}} / \mathrm{kg}_{\mathrm{H} 2}$ & [35] \\
\hline PV-EL capacity ratio & 2 & - & [32] \\
\hline PV LCOE & 51 & $€ / \mathrm{MWh}_{\mathrm{e}}$ & [36] \\
\hline $\begin{array}{c}\text { PV LCOE - CAPEX } \\
\text { share }\end{array}$ & $75 \%$ & - & [37] \\
\hline $\begin{array}{l}\text { Grid electricity } \\
\text { purchase cost }\end{array}$ & 150 & $€ / \mathrm{MWh}_{\mathrm{e}}$ & Assumed \\
\hline $\begin{array}{l}\text { PV surplus electricity } \\
\text { sale price }\end{array}$ & 30 & $€ / \mathrm{MWh}_{\mathrm{e}}$ & Assumed \\
\hline $\begin{array}{l}\text { SMR-CCS H} \\
\text { production cost }\end{array}$ & 1.9 & $€ / \mathrm{kg}_{\mathrm{H} 2}$ & [38] \\
\hline $\begin{array}{l}\text { Liquid } \mathrm{H}_{2} \text { truck } \\
\text { CAPEX }\end{array}$ & 207 & $€ / \mathrm{kg}_{\mathrm{H} 2}$ & [39] \\
\hline $\begin{array}{l}\text { Liquid } \mathrm{H}_{2} \text { truck } \\
\text { capacity }\end{array}$ & 4.3 & $\mathrm{t}_{\mathrm{H} 2} /$ vehicle & [39] \\
\hline $\begin{array}{c}\text { Compressed } \mathrm{H}_{2} \text { truck } \\
\text { CAPEX }\end{array}$ & 355 & $€ / \mathrm{kg}_{\mathrm{H} 2}$ & [40] \\
\hline $\begin{array}{l}\text { Compressed } \mathrm{H}_{2} \text { truck } \\
\text { capacity }\end{array}$ & 1 & $\mathrm{t}_{\mathrm{H} 2} /$ vehicle & [40] \\
\hline $\begin{array}{c}\text { Gaseous } \mathrm{H}_{2} \text { pipeline } \\
\text { CAPEX }\end{array}$ & $\begin{array}{r}4 \cdot 10^{3} \\
\mathrm{~W}\end{array}$ & $\begin{array}{l}A+336 € / \mathrm{km}, \\
\mathrm{h} \mathrm{A} \text { in } \mathrm{m}^{2}\end{array}$ & [5] \\
\hline
\end{tabular}

*Includes stack replacement after 10 years 


\section{Comparison and discussion}

The study investigates four cases, considering, at first, a mono-modality simulation for each of the three included transport technologies, and, then, a complete multimodality simulation that optimizes the selection of the transport technology. All simulations are performed with a workstation equipped with an Intel Xeon W-2123 $3.6 \mathrm{GHz}$ processor and $32 \mathrm{~GB}$ RAM. Due to the high model complexity and size that result from the detailed spatial and temporal resolutions, the integer variables representing the number of travelling trucks are relaxed to continuous values. With this adjustment, the monomodality simulations are each completed in about 10 minutes, whereas 43 hours are required for the multimodality case, thus proving the necessity of such a simplification to comply with the available resources.

As Figure 3 shows, the multi-modality simulation (labelled "Multi") yields the lowest average cost of hydrogen $\left(5.83 € / \mathrm{kg}_{\mathrm{H} 2}\right)$, while all mono-modality configurations (labelled "Mono -" with a suffix indicating the mode type) are more expensive, with values $4-11 \%$ higher. The multi-modality infrastructure exploits all the three transport technologies, with a predominance of pipeline and liquid hydrogen truck delivery, which meet the demand of 173 and 171 stations, respectively, while the remaining 22 receive compressed hydrogen via truck. Although the number of stations supplied via pipeline is almost the same as those that receive hydrogen via liquid hydrogen trucks, pipeline delivery accounts for more than $60 \%$ of the total hydrogen consumption, whereas liquid hydrogen represents only $25 \%$. This result is due to the fact that most of the stations supplied via liquid hydrogen truck are located in the southern provinces of the region (see Figure 4), which are characterised by a lower demand than the central provinces that are supplied via pipeline.

The comparison of the cost breakdown of the monomodality simulations (see Figure 3 ) shows the advantages and drawbacks of the different transport technologies. The share of the transport cost is considerably higher for pipeline and compressed hydrogen truck delivery. As far as the former is concerned, the large expense is due to the high investment costs required for pipeline installation, while the cost item 'Transport' in the case of compressed $\mathrm{H}_{2}$ truck delivery also covers the storage expenditure at demand nodes, since storage is performed with the same trailers that are used for transport. On the other hand, liquid hydrogen truck delivery benefits from lower specific costs for both transport and storage (see Table 1), but liquefaction is more capital and energy intensive than compression, leading to higher investment and operational cost, and, thus, to a significantly higher conditioning share. Production costs are slightly less impacting in the Mono-LT configuration, as the lower storage cost allows to better exploit PV plants, minimizing the purchase of grid electricity.

In the multi-modality simulation, the advantages of each technology are exploited, and each performs the tasks it is most suited for, yielding the overall lowest-cost infrastructure configuration. The cost breakdown is compared to that of the mono-modality configurations in Figure 3. In the multi-mode, since liquid hydrogen is employed to satisfy only a fraction of the total demand, the higher cost and consumption of liquefaction are compensated by the favourable economics of liquid transport and storage, as the combined share of production and conditioning is in line with those of the Mono-GP and Mono-GT scenarios, while the transport and storage at HRS costs are lower.

As opposed to all mono-modality configurations, the multi-modality infrastructure exploits four intermediate storage sites, with capacity of 1000, 1000, 405, and 183 $\mathrm{t}_{\mathrm{H} 2}$, respectively, which absorb the hydrogen overproduction from PV-fed electrolysis systems during summer to redistribute it in periods of lower solar radiation. The selected storage form is liquid due to the much lower investment costs, despite the boil-off. Such option is not exploited in the Mono-LT configuration, since, in the analysed case study, it becomes economically favourable when the production of liquid hydrogen is limited to few plants, as in the multi-modality case, in order to maximise its contribution.

By looking at the exploited transport networks (Figure 4 ), it emerges that the installed pipelines cover most of the region, consistently with the fact they are suitable to deliver large quantities over long distances, while compressed hydrogen trucks travel along a less extensive network and supply a limited number of stations, confirming that this transport modality is appropriate to meet relatively small delivery requirements.

In all the cases, the highest-share cost item is production, which is strongly based on PV-fed electrolysis systems, which account for about $95 \%$ of the delivered hydrogen quantities. In the production cost, the impact of the investment is nearly twice the operational expenditure, and all the infrastructure configurations feature a similar proportion.

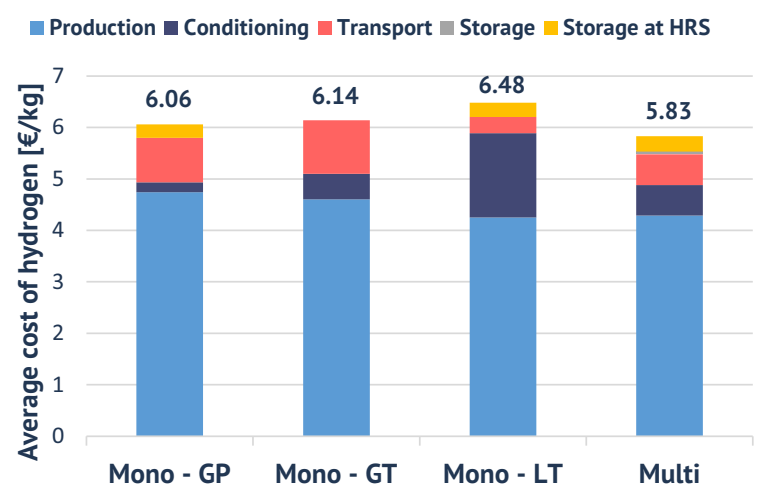

Figure 3. Average cost of hydrogen with cost breakdown for the different configurations. In the 'Mono-GT' case, the item 'Transport' covers also the storage needs at demand sites. 


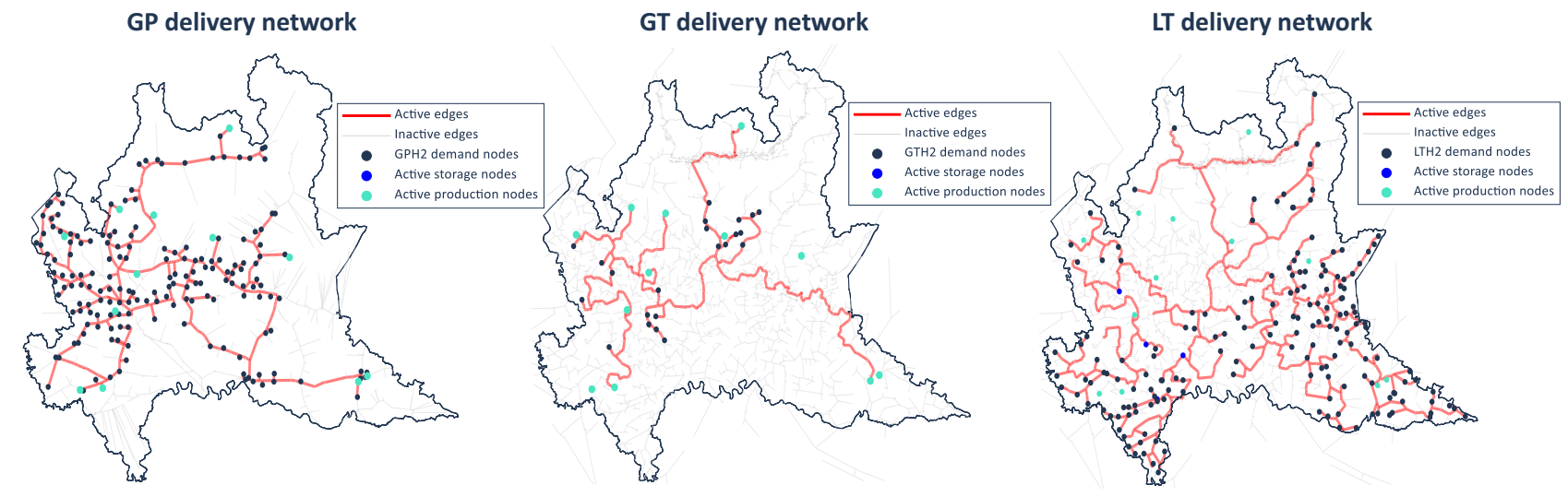

Figure 4. Optimized transport network for each transport technology in the multi-modality configuration.

\section{Conclusions}

By means of an optimization model of the hydrogen supply chain, this study investigated the infrastructural needs related to the widespread adoption of hydrogen as a fuel for clean mobility, focusing on the comparison between the mono- and multi-modality formulations. The former consider a single transport technology at a time, while the latter optimizes the choice of the transport technology within the overall system simulation.

The analysis developed as an example the case study of the Italian region of Lombardy, considering a longterm scenario (2050) featuring a distributed demand of hydrogen for FCEVs, accounting for $25 \%$ of the passenger car stock. The study considered four simulations, comparing three mono-modality scenarios in which either hydrogen pipelines, compressed hydrogen trucks, or liquid hydrogen trucks are adopted separately, and a multi-modality configuration in which the three transport modalities are allowed together and their selection is optimized. Results showed that the multimodality infrastructure yields the lowest average cost of hydrogen delivered to refuelling stations, with a value of $5.83 € / \mathrm{kg}_{\mathrm{H} 2}$, whereas the mono-modality cases are $4 \%$ $11 \%$ more expensive. The multi-modality configuration simultaneously exploits all the three included technologies, with $47.3 \%$ of stations supplied via pipeline, $46.7 \%$ via liquid hydrogen truck, and $6.0 \%$ via compressed hydrogen truck.

Beyond the specific case study, the model application proves the relevance of the multi-modality formulation, showing that the multiplicity of transport options cannot be disregarded when modelling the HSC, as the costoptimal alternative cannot be predicted a priori and the cheapest solution is likely a combination of different modalities. On the other hand, the multi-modality formulation significantly increases the model complexity, as, in the case study, the solution is obtained in dozens of hours, whereas mono-modality simulations require few minutes. Accordingly, conventional computational resources become no longer suitable to dealing with a wider spatial scale (e.g., considering a national case study) or with a broader set of transport modalities (e.g., including also liquid organic hydrogen carriers or shipping) while maintaining the proposed model detail, thus requiring to look into high-performance computing clusters for applications of increasing size.

Further analyses will investigate hydrogen storage at intermediate sites, evaluating the cost impact and the required cost reduction to attain overall advantages, e.g., by considering alternative technologies such as underground storage in lined rock caverns, which could provide cheaper seasonal storage and, thus, a better integration with RES-based hydrogen production.

\section{References}

[1] European Commission, "European Green Deal," 2020. https://ec.europa.eu/info/strategy/priorities-20192024/european-green-deal_en.

[2] Fuel Cells and Hydrogen Joint Undertaking (FCH), "Hydrogen Roadmap Europe," 2019.

[3] IEA, "Europe: key energy statistics." https://www.iea.org/regions/europe.

[4] G. Guandalini and S. Campanari, "Well-to-wheel driving cycle simulations for freight transportation: Battery and hydrogen fuel cell electric vehicles," 2018 Int. Conf. Electr. Electron. Technol. Automotive, Automot. 2018, no. ii, pp. 0-5, 2018.

[5] C. Yang and J. Ogden, "Determining the lowestcost hydrogen delivery mode," Int. J. Hydrogen Energy, vol. 32, no. 2, pp. 268-286, 2007.

[6] S. Baufumé et al., "GIS-based scenario calculations for a nationwide German hydrogen pipeline infrastructure," Int. J. Hydrogen Energy, vol. 38, no. 10, pp. 3813-3829, 2013.

[7] H. Talebian, O. E. Herrera, and W. Mérida, "Spatial and temporal optimization of hydrogen fuel supply chain for light duty passenger vehicles in British Columbia," Int. J. Hydrogen Energy, vol. 44, no. 47, pp. 25939-25956, 2019.

[8] N. Strachan, N. Balta-Ozkan, D. Joffe, K. McGeevor, and N. Hughes, "Soft-linking energy systems and GIS models to investigate spatial hydrogen infrastructure development in a lowcarbon UK energy system," Int. J. Hydrogen Energy, vol. 34, no. 2, pp. 642-657, 2009.

[9] P. Colbertaldo, S. Cerniauskas, T. Grube, M. 
Robinius, D. Stolten, and S. Campanari, "Clean mobility infrastructure and sector integration in long-term energy scenarios: The case of Italy," Renew. Sustain. Energy Rev., vol. 133, p. 110086, 2020.

[10] L. Li, H. Manier, and M. A. Manier, "Integrated optimization model for hydrogen supply chain network design and hydrogen fueling station planning," Comput. Chem. Eng., vol. 134, 2020.

[11] A. Almansoori and A. Betancourt-Torcat, "Design of optimization model for a hydrogen supply chain under emission constraints - A case study of Germany," Energy, vol. 111, pp. 414429, 2016.

[12] S. Samsatli, I. Staffell, and N. J. Samsatli, "Optimal design and operation of integrated wind-hydrogen-electricity networks for decarbonising the domestic transport sector in Great Britain," Int. J. Hydrogen Energy, vol. 41, no. 1, pp. 447-475, 2016.

[13] A. Ochoa Bique and E. Zondervan, "An outlook towards hydrogen supply chain networks in 2050 - Design of novel fuel infrastructures in Germany," Chem. Eng. Res. Des., vol. 134, pp. 90-103, 2018.

[14] M. Moreno-Benito, P. Agnolucci, and L. G. Papageorgiou, "Towards a sustainable hydrogen economy: Optimisation-based framework for hydrogen infrastructure development," Comput. Chem. Eng., vol. 102, pp. 110-127, 2017.

[15] J. Kim, Y. Lee, and I. Moon, "Optimization of a hydrogen supply chain under demand uncertainty," Int. J. Hydrogen Energy, vol. 33, no. 18, pp. 4715-4729, 2008.

[16] M. Dayhim, M. A. Jafari, and M. Mazurek, "Planning sustainable hydrogen supply chain infrastructure with uncertain demand," Int. J. Hydrogen Energy, vol. 39, no. 13, pp. 6789-6801, 2014.

[17] W. Won, H. Kwon, J. H. Han, and J. Kim, "Design and operation of renewable energy sources based hydrogen supply system: Technology integration and optimization," Renew. Energy, vol. 103, pp. 226-238, 2017.

[18] L. Welder, D. S. Ryberg, L. Kotzur, T. Grube, M. Robinius, and D. Stolten, "Spatio-temporal optimization of a future energy system for powerto-hydrogen applications in Germany," Energy, vol. 158, pp. 1130-1149, 2018.

[19] A. Ochoa Bique, L. K. K. Maia, F. La Mantia, D. Manca, and E. Zondervan, "Balancing costs, safety and $\mathrm{CO} 2$ emissions in the design of hydrogen supply chains," Comput. Chem. Eng., vol. 129, 2019.

[20] N. Sabio, A. Kostin, G. Guillén-Gosálbez, and L. Jiménez, "Holistic minimization of the life cycle environmental impact of hydrogen infrastructures using multi-objective optimization and principal component analysis," Int. J. Hydrogen Energy, vol. 37, no. 6, pp. 5385-5405, 2012.

[21] Z. Li, D. Gao, L. Chang, P. Liu, and E. N.
Pistikopoulos, "Hydrogen infrastructure design and optimization: A case study of China," Int. J. Hydrogen Energy, vol. 33, no. 20, pp. 5275-5286, 2008 .

[22] S. De-León Almaraz, C. Azzaro-Pantel, L. Montastruc, and M. Boix, "Deployment of a hydrogen supply chain by multi-objective/multiperiod optimisation at regional and national scales," Chem. Eng. Res. Des., vol. 104, pp. 1131, 2015.

[23] G. Guillen-Gosalbez, F. D. Mele, and I. E. Grossman, "A Bi-Criterion Optimization Approach for the Design and Planning of Hydrogen Supply Chains for Vehicle Use," AIChE J., vol. 56, no. 3, pp. 650-667, 2010.

[24] J. H. Han, J. H. Ryu, and I. B. Lee, "Multiobjective optimization design of hydrogen infrastructures simultaneously considering economic cost, safety and CO2 emission," Chem. Eng. Res. Des., vol. 91, no. 8, pp. 1427-1439, 2013.

[25] J. O. Robles, C. Azzaro-Pantel, and A. AguilarLasserre, "Optimization of a hydrogen supply chain network design under demand uncertainty by multi-objective genetic algorithms," Comput. Chem. Eng., vol. 140, 2020.

[26] M. Reuß, T. Grube, M. Robinius, and D. Stolten, "A hydrogen supply chain with spatial resolution: Comparative analysis of infrastructure technologies in Germany," Appl. Energy, vol. 247, no. December 2018, pp. 438-453, 2019.

[27] C. Wulf et al., "Life Cycle Assessment of hydrogen transport and distribution options," $J$. Clean. Prod., vol. 199, pp. 431-443, 2018.

[28] F. Parolin, P. Colbertaldo, and S. Campanari, "Design and Optimization of a Multi-Mode Hydrogen Delivery Infrastructure for Clean Mobility," accepted paper SDEWES2021.0753 at 16th Conference on Sustainable Development of Energy, Water, and Environment Systems (SDEWES2021), Dubrovink, Croatia, Oct 2021.

[29] ESRI, "ESRI Shapefile Technical Description," 1998.

[30] P. Colbertaldo, "Power-to-hydrogen for longterm power and transport sector integration," Politecnico di Milano, 2019.

[31] Il Sole 24 Ore, "Le raffinerie e l'indotto arrancano," 2014. https://www.infodata.ilsole24ore.com/2014/07/3 1/le-raffinerie-e-lindotto-arrancano/.

[32] P. Colbertaldo, G. Guandalini, G. Lozza, and S. Campanari, "Sizing of integrated solar photovoltaic and electrolysis systems for clean hydrogen production," in EFC2019 European Fuel Cell Technology \& Applications - Piero Lunghi Conference EFC2019, 2019, pp. 20192020.

[33] European Commission, "Eurostat." https://ec.europa.eu/eurostat/home.

[34] United States Department of Labor, "U.S. Bureau of Labor Statistics.” https://www.bls.gov/. 
[35] IRENA, "Hydrogen From Renewable Power," 2018.

[36] W. Zappa, M. Junginger, and M. van den Broek, "Is a $100 \%$ renewable European power system feasible by 2050?," Appl. Energy, vol. 233-234, no. November 2018, pp. 1027-1050, 2019.

[37] IRENA, "Renewable Power Generation Costs in 2018," 2019.

[38] IEAGHG, "Techno - Economic Evaluation of SMR Based Standalone (Merchant) Hydrogen
Plant with CCS," 2017.

[39] M. Reuß, T. Grube, M. Robinius, P. Preuster, P. Wasserscheid, and D. Stolten, "Seasonal storage and alternative carriers: A flexible hydrogen supply chain model," Appl. Energy, vol. 200, pp. 290-302, 2017.

[40] Fuel Cells and Hydrogen 2 Joint Undertaking (FCH JU), "Addendum to the Multi-Annual Work Plan 2014-2020,” 2018. 